\title{
An Evolving Expert System for Maize Plant Nutrient Deficiency using Image Processing Technique
}

\author{
S. Sridevy ${ }^{1}$, Dr. Anna Saro Vijendran ${ }^{2}$ \\ Assistant Professor, Department of PS and IT, AEC and RI, Tamilnadu Agricultural University, Tamilnadu, India \\ ${ }^{2}$ Director \& Head, Department of MCA in SNR Sons College, Coimbatore, Tamil Nadu, India
}

\begin{abstract}
Maize is an important cereal crop grown widely throughout the world in a range of agro-ecological environments. The crop can be used for food and non-foods products. Nonetheless, despite the nutritional and economic significance of the maize crop, the diseases incidence on maize plantations is fast becoming a constraint in farmers' quest for a bountiful harvest. The efforts of agricultural extension agents seem not to be sufficient in tackling this menace since there is always a limit to how far the human capacity can be stretched in the face of highly demanding situations. Hence, this paper proposed expert system which infers nutrient deficiency of maize crop namely Nitrogen, Phosphorus and potassium using Multivariate Image Analysis and Multivariate Partial Least Square Approach. The expert system would provide immediate and instant information to the possible disease affecting the life of maize with the consideration of several known symptoms supplied. It would fast-track information service delivery on the part of the large-scale industrial grain farmers which make use of it for emergency situations of disease outbreak on the farm, pending the arrival of the agricultural extension agent.
\end{abstract}

Keywords: maize, deficiency, expert, agriculture and nitrogen

\section{Introduction}

Maize (Zea mays) which is known in many English-speaking countries as corn is a grain domesticated by indigenous peoples in Mesoamerica in prehistoric times. The Aztecs and Mayans cultivated it in numerous varieties throughout central and southern Mexico, to cook or grind in a process called nixtamalization. Later the crop spread through much of the Americas. Between 1250 and 1700, nearly the whole continent had gained access to the crop. Any significant or dense populations in the region developed a great trade network based on surplus and varieties of maize crops. After European contact with the Americas in the late 15th and early 16th centuries, explorers and traders carried maize back to Europe and introduced it to other countries through trade [1]. Maize spread to the rest of the world due to its popularity and ability to grow in diverse climates thus explaining why it is one of the major staple food crops consumed in today's Nigerian society. Maize is a cereal crop that is grown widely throughout the world. More maize is produced annually than any other grain. About 50 species exist and consist of different colors, textures and grain shapes and sizes. The white and yellow varieties are preferred by most people depending on the region [2]. Maize is the third most important food grain in India after wheat and rice. In India, about $28 \%$ of maize produced is used for food purpose, about $11 \%$ as livestock feed, $48 \%$ as poultry feed, $12 \%$ in wet milling industry (for example starch and oil production) and $1 \%$ as seed (AICRP on Maize, 2007) [4] . In the last one decade, it has registered the highest growth rate among all food grains including wheat and rice because of newly emerging food habits as well as enhanced industrial requirements Sequel to the nutritional and economic significance of the maize crop, it is noteworthy to mention the fact that diseases incidence on maize plantations is fast becoming a constraint in farmers' quest for a bountiful harvest from their maize farms and efforts of agricultural extension agents seem not to be sufficient in tackling this menace. Maize diseases include but are not limited to downy mildew, rust, leaf blight, stalk and ear rots, leaf spot, and maize streak virus [3]. The Expert System for diagnosis and treatment of maize diseases is therefore a software package designed to further enable efficiency and effectiveness in maize diseases management in terms of diagnosis and treatment as required by agricultural extension agents and other end-users (large-scale maize industrial farmers) to enhance higher productivity.

\section{Overview of Expert Systems}

Expert systems are most valuable to organizations that have a high-level of know-how experience and expertise that cannot be easily transferred to other members. They are designed to carry the intelligence and information found in the intellect of experts and provide this knowledge to other members of the organization for problem-solving purposes [5]. Expert systems capture scarce expert knowledge and render it archival. This is an advantage when losing the expert would be a significant loss to the organization. Distributing the expert knowledge enhances employee productivity by offering necessary assistance to make the best decision. Improvements in reliability and quality frequently appear when expert systems distribute expert advice, opinion, and explanation on demand. Expert systems are capable of handling enormously complex tasks and activities as well as an extremely rich knowledge-database structure and content. As such, they are well suited to model human activities and problems. Expert systems can reduce production downtime 


\section{International Journal of Science and Research (IJSR) \\ ISSN (Online): 2319-7064}

Index Copernicus Value (2013): 6.14 | Impact Factor (2014): 5.611

and, as a result, increase output and quality. Additionally, expert systems facilitate the transfer of expertise to remote locations using digital communications. In specific situations, ongoing use of an expert system may be cheaper and more consistent than the services of a human expert [6]. Expert systems can track production variables, tabulate statistics, and identify processes that don't match the expected patterns, signaling potential problems. Moreover, integrated expert systems can immediately notify the appropriate person to correct a problem in the manufacturing process [7]. Compared to traditional programming techniques, expert system approaches provide the added flexibility and easier modifiability with the ability to model rules as data rather than as code. In situations where an organization's IT department is overwhelmed by a software-development backlog, rule-engines, by facilitating turnaround, provide a means that can allow organizations to adapt more readily to changing needs. In practice, modern expert-system technology is employed as an adjunct to traditional programming techniques, and this hybrid approach allows the combination of the strengths of both approaches. Thus, rule engines allow control through programs and user interfaces written in a traditional language, and also incorporate necessary functionality such as inter-operability with existing database technology.

Characteristics of Agricultural Expert System:

- It simulates human reasoning about a problem domain, rather than simulating the domain itself.

- It performs reasoning over representations of human knowledge

- It solves problems by heuristic or approximate methods

\section{Literature Survey}

\subsection{Expert System for Plant Disease Diagnose}

Naser, Kashkash and M. Fayyad designed and developed in expert system with two different methods for diagnosing plant diseases. The first one is using the descriptive method (step by step) and the other one is the graphical representation method. A preliminary evaluation of the system showed that the expert system with the graphical representation is more favorable than the descriptive one. This is due to the difficulties in describing the symptoms of the disease. This is due to the difficulties in describing the symptoms of the disease. On the other hand, a graphical picture of the symptoms does not require much description from the user. Expert Systems are considered one of the most successful methods used to help and support users in making the right decisions. Present expert systems saved a lot of time and effort in identifying plant disease due to the mechanism used in receiving the data and providing the decisions. [9]

\subsection{A Web-based Distance Diagnostic and identification system}

Xin,Zazueta and Beck presented a Web-based Distance Diagnostic and Identification System (DDIS), developed at the University of Florida. County extension agents were trained to submit field data and digital media of pest, plant disease, insect, animal, weed, management, and plant nutrient problems to specialists statewide for rapid diagnosis through Internet. The system provided an effective collaboration environment for extension agents and specialists to share information on pest, plant disease, insect, and plant management problems. Specialists around the state can perform distance diagnosis and make recommendations to extension agents. Through direct interaction with specialists, county agents become more familiar with plant disease problems. Turnaround time was reduced from days to hours. [10]

\subsection{Expert System for Crop Production Management}

Rafea, El-Azhari and Hassan presented a general framework for integrating crop management expert systems with multimedia. The experience of integrating images with the expert system of cucumber has revealed the implementation problems to be addressed when images are to be enclosed in the expert system. These problems can be classified according to the steps followed for integrating the images with the expert system namely: images identification, collection and scanning of images, and knowledge base modification. Studying, the presentation of observation into images, and identifying these images are very important in order to save efforts in collecting, scanning, and storing images which may not be used later. [11]

\subsection{Integrating Agricultural Expert System with Databases and Multimedia}

Rafea revealed in this paper that integration of expert system with data bases is needed to store the static data of a certain plantation. The main technical problem that can be raised due to integration with data bases is the maintenance of both the knowledge base and the data base assuming that the expert system tools supports calling a data base retrieval program. The unavailability of a such retrieval program is a major problem which should be taken care of from the very beginning of an expert system project. The maintenance problem could be solved, either manually in case that the developer uses ready made package, by taking care of the data base, when making any modification to the knowledge base, or by building a knowledge and a data base management system in case that the developer uses a tool built in house.

The needs for integration with multimedia are: the enhancement of the symptoms acquisition, disorder verification and the explanation of agriculture operations. The main problem identified to accomplish this integration is the proper identification of the images, video tapes to be integrated, and the knowledge modification to link the different attributes. [12]

\subsection{Natural Resources Conservation and Crop management Expert System}

Rafea presented in this paper that how expert systems for crop management can help in natural resources conservation. This is done through giving an overview of the status of natural resources in Egypt with emphasis on water and soil 


\section{International Journal of Science and Research (IJSR) \\ ISSN (Online): 2319-7064}

Index Copernicus Value (2013): 6.14 | Impact Factor (2014): 5.611

resources, describing briefly five expert systems developed for managing cucumber, tomato, orange, lime, and wheat, and explaining how the recommendations that optimize the output relative to the agricultural inputs will lead to environmental conservation as it will be guaranteed that no extra inputs will be provided such as water, fertilizers and pesticides without a return in the yield. The paper also responds to the issue concerning the integration with other types of software and presents how decision makers at different levels can use crop management expert systems. [13]

\subsection{Expert System for Leaf Disease Detection and Diagnosis}

El- Helly, Rafea and El- Gammal developed an integrated image processing expert system capable of diagnosing three disorders, Downy mildew with percentage 84\%, Leafminer with percentage $74 \%$, and Powdery mildew with percentage $94 \%$. Also, the system is capable of deciding the normal leaves with a percentage $98 \%$. Moreover, the system is capable to recognizing the unknown disorder with a percentage $92 \%$. A set of features was selected to be extracted using feature extraction phase, and those features were stored in the feature database, which is designed for this purpose. [14]

\subsection{An Automated System for Plant Leaf Recognition}

Chaki and Parekh proposed an automated system for recognizing plant species based on leaf images. Plant leaf images corresponding to three plant types, are analyzed using three different shape modelling techniques, the first two based on the Moments-Invariant (M-I) model and the Centroid-Radii (C-R) model and the third based on a proposed technique of Binary-Superposition (B-S). For the M-I model the first four central normalized moments have been considered. For the C-R model an edge detector has been used to identify the boundary of the leaf shape and 36 radii at 10 degree angular separation have been used to build the shape vector. The proposed approach consists of comparing binary versions of the leaf images through superposition and using the sum of non-zero pixel values of the resultant as the feature vector. The data set for experimentations consists of 180 images divided into training and testing sets and comparison between them is done using Manhattan, Euclidean and intersection norms [15].

\subsection{Integrating Diagnostic Expert System with Image Processing}

El- Helly, Rafea,El-Gammal and El Whab demonstrated the usefulness of integrating an image analyzer within a diagnostic expert system model through a real life example. In order to diagnose a disorder from a leaf image, four image-processing phases were applied: enhancement, segmentation, feature extraction, and classification. In order to employ this system, the system was trained using a set of images of disorders. The system was tested on 3 cucumber disorders. The results of this test indicated that this system could indeed identify disorders with a high level of accuracy. Applying this model to any other crop disorders requires only special care to be taken in order to acquire a sufficient set of images representative of these disorders for use in the training step. Integrating this model within a diagnostic expert system then will greatly reduce any error prone dialogue between the system and the user while resulting in increased accuracy in the systems diagnosis. [16]

\subsection{Image Based Rapeseed-Mustard Disease Expert System}

Kumar, Lehri, Sharma, Meena and Kumar developed Image based Rapeseed-Mustard Disease expert system" which is an integration of image and textual data. The system can be used by extension personnel, researchers and farmers to identify rapeseed-mustard diseases and enable their management. User can easily identify the disease on the bases for photos of symptoms and text description of disease. The user friendly software developed using windowing environment, thus provides enough facilities to identify the disease and to suggest the remedy conveniently. [17]

\subsection{Review on Methodologies used for Development of Expert Systems}

Wide varieties of methodologies are available for designing expert systems [8].

\section{a) Rule Based System:}

The knowledge base is a collection of rules or other information provided by the human expert. These rules consist of a condition or premise followed by an action or conclusion (IF condition...THEN action). The rule can then be used to perform operations on data given as input in order to reach appropriate conclusion.

\section{b) Case Based Reasoning}

In this approach, knowledge base contains the solutions that have been already achieved uses to get a solution to the new problem. Here the, descriptions of past experience of human experts, represented as cases, are stored in a database for later retrieval when the user encounters a new case with similar parameters

\section{c) Knowledge based system}

The concept of KBS is rooted in the field of artificial intelligence. This system tries to initiate and adapt human knowledge in a computer program. The basic components of KBS are knowledgebase, inference engine, tool for knowledge engineering, and specific user interface.

\section{d) Neural networks}

The concept artificial neural network (ANN) is used to implement software simulations for the massively parallel processes that involve processing elements interconnected in network architecture. It can be used to convert data signals to special format

\section{e) Fuzzy Logic}

Which handles the issues with uncertainty? This technique uses the theory of fuzzy sets, simulates the normal human reasoning process by allowing the computer to behave less precisely and logically than conventional computers

\section{Volume 5 Issue 2, February 2016}




\section{International Journal of Science and Research (IJSR) \\ ISSN (Online): 2319-7064}

Index Copernicus Value (2013): 6.14 | Impact Factor (2014): 5.611

\section{f) Object oriented methodology}

This method combines one object with specific procedure that operates on data. It is mainly used for disease diagnosis.

\section{g) Modeling}

It builds a logical model design for all problem domains. It provides quantitative methods to represent and acquire knowledge so that it can provide great platform for expert system development.

\section{i) Ontology}

Ontology can be treated as system of vocabulary. Ontology is used to describe problem and domain knowledge, It can act as a communication link between human experts and knowledge engineer.

Main methodologies used for agricultural applications are shown in Table [1]

\begin{tabular}{|l|l|}
\multicolumn{2}{c}{ Table (1) } \\
\hline Methodology & $\begin{array}{l}\text { Applications in Agriculture field } \\
\text { diseases } \\
\text { Agricultural advisor } \\
\text { Production planning }\end{array}$ \\
\hline Knowledge based system & $\begin{array}{l}\text { Agricultural management } \\
\text { Plant process control } \\
\text { Environmental protection }\end{array}$ \\
\hline Fuzzy based system & $\begin{array}{l}\text { Uncertainty reasoning } \\
\text { Disease diagnosis }\end{array}$ \\
\hline $\begin{array}{l}\text { Neural network based } \\
\text { system }\end{array}$ & $\begin{array}{l}\text { Decision making } \\
\text { Knowledge learning }\end{array}$ \\
\hline Ontology & $\begin{array}{l}\text { Agricultural decision support, } \\
\text { crop protection }\end{array}$ \\
\hline
\end{tabular}

\section{Design and Development of Proposed Expert System}

Identification of maize crop deficiency is the task handled by plant pathologists in various institutions where agriculture is studied as a subject. The farmers are not capable enough to identify the symptoms of the leaf diseases of the cereals. They can recognize the common disease symptoms only. Identification of leaf diseases of crops and the other related information is totally dependent on the experts. This process is avoids time consuming and the valuable time of farmers not get wasted. In this proposed work the image processing is taken as a tool to determine the deficiency of maize crop with the two different approaches Multivariate Image Analysis \& Multivariate Partial Least Square. Initially, the images of maize crop leaves are obtained using a digital camera then image preprocessing, feature extraction and classification of leaf is done under this inference part.

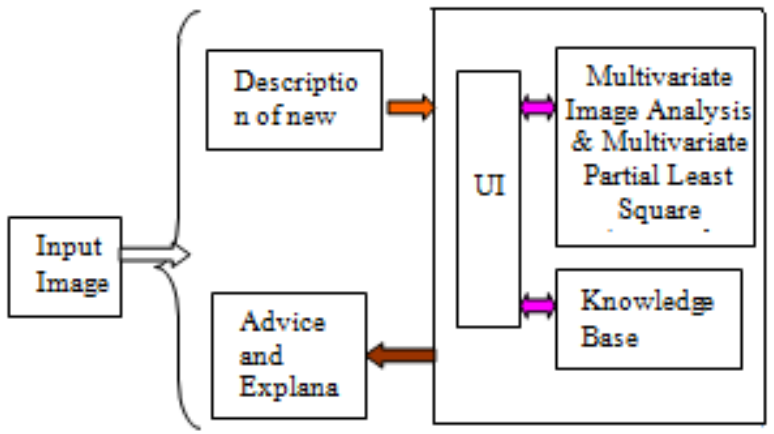

Figure 1: Proposed Maize Expert System Architecture
From the figure 1 it shows three major components of an expert system they are as follows:

- The knowledge base is the collection of facts and rules which describe all the knowledge about the problem domain.

- The inference engine is the part of the system that chooses which facts and rules to apply when trying to solve the users query which is achieved by two approaches namely Multivariate Image Analysis \& Multivariate Partial Least Square Approach

- The user interface is the part of the system which takes in the users query in a readable form and passes it to the inference engine.

- It then displays the advice as the results to the farmers about the type of nutrient deficiency affected in the maize crop leaf.

In this model, the defected image of defected maize leaf is used as an input to the model. The image analyzer components detect the abnormal symptom in the defected image then extract their features and classify those features to specific nutrition deficiency.

\section{Proposed Expert System Working Model}

The phases of proposed Expert System are discussed as follows:

\subsection{Colour Transformation Structure Phase}

First, the RGB images of leaves are converted into Hue Saturation Intensity (HSI) colour space representation. The purpose of the colour space is to facilitate the specification of colours in some standard, generally accepted way. HIS (hue, saturation, intensity) colour model is a popular colour model because it is based on human perception.

Electromagnetic radiation in the range of wavelengths of about 400 to 700 nanometers is called visible light because the human visual system is sensitive to this range. Hue is generally related to the wavelength of a light Hue is a colour attribute that refers to the dominant colour as perceived by an observer. Saturation refers to the relative purity or the amount of white light added to hue and intensity refers to the amplitude of the light. Colour spaces can be converted from one space to another easily. After the transformation process, the $\mathrm{H}$ component is taken into account for further analysis. S and I are dropped since it does not give extra information.

- Masking green pixels: In this step, we identify the mostly green coloured pixels. After that, based on specified and varying threshold value that is computed for these pixels using Otsu's method, these mostly green pixels are masked as follows: if the green component of pixel intensities is less than the pre-computed threshold value, the red, green and blue components of the this pixel is assigned to a value of zero. This is done in sense that these pixels have no valuable weight to the disease identification and classification steps, and most probably those pixels represent healthy areas in the leave. Furthermore, the 


\section{International Journal of Science and Research (IJSR) \\ ISSN (Online): 2319-7064}

Index Copernicus Value (2013): 6.14 | Impact Factor (2014): 5.611

image processing time should become significantly reduced. In next step the pixels with zero red, green and blue values were completely removed.

- This phase is helpful as it gives more accurate disease classification and identification results with satisfied performance and the overall computation time should become significantly less.

\subsection{Histogram Equalization for Image Enhancement}

Histogram Equalization is a technique that generates a gray map which changes the histogram of an image and redistributing all pixels values to be as close as possible to a user -specified desired histogram. HE allows for areas of lower local contrast to gain a higher contrast. Histogram equalization automatically determines a transformation function seeking to produce an output image with a uniform Histogram. Histogram equalization is a method in image processing of contrast adjustment using the image histogram. This method usually increases the global contrast of many images, especially when the usable data of the image is represented by close contrast values. Through this adjustment, the intensities can be better distributed on the histogram. Histogram equalization accomplishes this by effectively spreading out the most frequent intensity values. Suppose that we observe a m-dimensional zero mean input signal at time $t$, where, means the transposition of matrices and vectors. Then the $n$-dimensional whitening signal, $x(t)$, is given by the following equation:

$$
x(t)=M v(t)=D^{-1 / 2} E^{\prime} v(t)
$$

Where $M$ means a $n \times m(n \leq m)$ whitening matrix that is given by a matrix of eigen values, $\mathrm{D}$, and a matrix of eigenvectors, E. Here, assume that $v(t)$ is composed of $n$ statistically independent signals $\mathrm{s}(\mathrm{t})=\left\{\mathrm{s}_{1}(\mathrm{t}), \mathrm{s}_{2}(\mathrm{t}), . . \mathrm{s}_{\mathrm{n}}(\mathrm{t})\right\}$. Then, the following linear transformation from $\mathrm{x}(\mathrm{t})$ to $\mathrm{s}(\mathrm{t})$ exists:

$$
s(t)=W x(t)
$$

$\mathrm{W}=\left(\mathrm{W}_{1}, \mathrm{~W}_{2}, \mathrm{~W}_{\mathrm{n}}\right)$ is often called a separating matrix, and it can be acquired through the training of a two-layer feed forward neural network. This neural network has n outputs denoted asand the ith row vector, ofW corresponds to a weight vector from inputs to the ith output. The term 'independent' is used here according to the following definition in statistics:

$$
p\left[s_{1}(t), \ldots, s_{n}(t)\right]=\prod_{i=1}^{n} p i\left[s_{i}(t)\right]
$$

Where is a probability density function. Since the above probability density function is not preliminary unknown, suitable objective functions should be devised such that neural outputs are satisfied with Eq. (3) as much as possible, that . Karhunen and Oja have proposed the following objective function [10], $\mathrm{J}($.$) , to be maximized in terms of$ output signals:

$$
J(\hat{s})=\sum_{i=1}^{n}\left|E\left\{s_{i}^{\sim 4}\right\}-3\left[E\left\{s_{i}^{2}\right\}\right]^{2}\right|
$$

Where E\{.\} means expectation. As well known, Eq.(4) corresponds to the fourth-order cumulants of called kurtosis. Learning algorithms for a separation matrix, $\mathrm{W}$, are derived from the gradient of Eq. (20). In the followings, we adopt Fast ICA algorithm proposed by Hyvarinen \& Oja in which fixed points of the gradient are obtained on-line.

\subsection{Feature Extraction Phase}

- Energy: Energy also means uniformity, or angular second moment (ASM). The more homogeneous the image is, the larger the value. When energy equals to 1 , the image is believed to be a constant image.

$$
\text { Energy }=\sum_{i=1}^{N_{g}} \sum_{j=1}^{N_{g}} P_{d}^{2}(i, j)
$$

- Entropy: Entropy is a measure of randomness of intensity image

$$
\text { Entropy }=-\sum_{i=1}^{N_{g}} \sum_{i=1}^{N_{g}} P_{d}(i, j) \log \left(P_{d}(i, j)\right)
$$

\subsection{Detecting Maize Crop leaf Deficiency}

In this phase two methods are used multivariate image analysis and Multivariate Partial Least Square using the obtained feature extraction of maize leaves

\subsubsection{Multivariate image analysis:}

Principal Component Analysis (PCA) is the workhorse of MIA. The key is the proper reorganization (matricizing) of the original 3-way or higher array. Unfolding is done so that each pixel (or voxel) becomes a single row in the analysis. Thus an image that is originally I by $\mathrm{J}$ pixels with $\mathrm{K}$ spectral channels is reshaped to form a two way array that is IxJ by K. PCA can then be performed on this matrix in the usual way. Mean centering is typically done and in some circumstances variance scaling may be used. After the PCA model is calculated, the scores, residuals and $\mathrm{T} 2$ values can be folded back up to reform images. Loadings vectors can be interpreted in the usual way.

\subsubsection{Multivariate Partial Least Square (MPLS)}

Multivariate data analysis is the simultaneous observation of more than one characteristic. In contrast to the analysis of univariate data, in this approach not only a single variable or the relation between two variables can be investigated, but the relations between many attributes can be considered. In general consider an multivariate linear regression model with an $\mathrm{n} \times \mathrm{m}$ matrix $\mathrm{X}$ of predictors and an $\mathrm{n} \times \mathrm{p}$ matrix $\mathrm{Y}$ of dependent variables; both matrices are mean-centered 


\section{International Journal of Science and Research (IJSR) \\ ISSN (Online): 2319-7064}

Index Copernicus Value (2013): 6.14 | Impact Factor (2014): 5.611

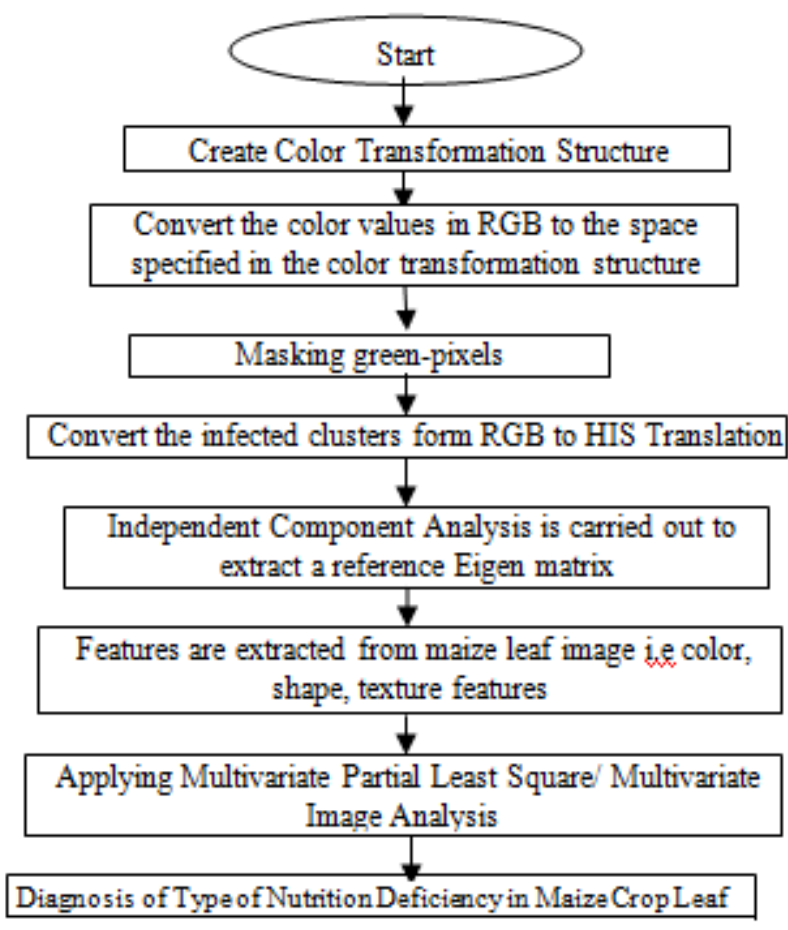

Figure 2: Proposed Expert System Working Model

\section{Results}

The proposed expert system is developed using java. Images of maize crop plant are collected from the Agricultural University, Coimbatore. The acquired leaf images are converted into HSI format. From the hue content, the cooccurrence features like, Cluster shade and Cluster prominence are derived. The feature sets are used for analysis type of nutrition deficiency. Result of some plant leaves are shown below. Some of the examples are shown below:

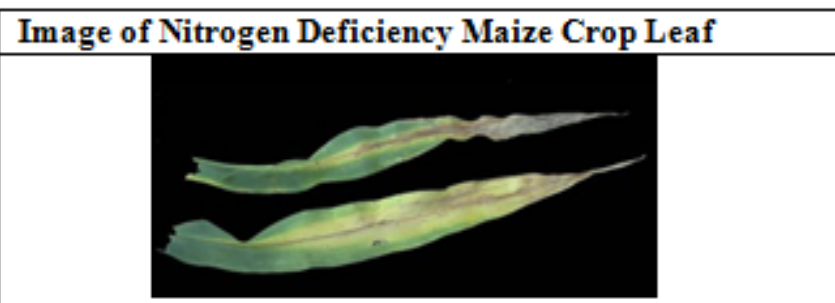

\section{Description}

Light green leaf and plant color with the older leaves tuming yellow, leaves that will eventually tum brown and die. Plant growth is slow; plants will be stunted, and will mature early.

Excess

Plants will be dark green in color and new growth will be succulent; susceptible if subjected to disease and insect infestation; and subjected to drought stress, plants will easily lodge. Blossom abortion and lack of fruit set will occur

\section{Ammonium toxicity}

Plants fertilized with ammonium-nitrogen (NH4- N) may exhibit ammonium-toxicity symptoms, with carbohydrate depletion andreduced plant growth. Lesions may occur on plant stems, there may be a downward cupping of the leaves, and a decay of the conductive tissue at the base of the stem with wilting of the plants under moisture stress. Blossom-nd rot of fruit will occur and $\mathrm{Mg}$ deficiency symptoms may also occur.
Figure 3 shows the Nitrogen Deficiency of maize crop leaf and the description about it to the users given by expert system

\begin{tabular}{l}
\hline Image of Phosphorus Deficiency Maize Crop Leaf \\
Description \\
Plant growth will be slow and stunted, and the older leaves \\
will have a purple coloration, particularly on the underside. \\
Excess \\
Phosphorus excess will not have a direct effect on the plant \\
but may show visual deficiencies of Zn, Fe, and Mn. High P \\
may also interfere with the normal Ca nutrition, with typical \\
Ca deficiency symptoms occurring.
\end{tabular}

Figure 4 shows the Phosphorus Deficiency of maize crop leaf and the description about it to the users given by expert system

\begin{tabular}{l}
\hline Image of Potassium Deficiency Maize Crop Leaf \\
Description \\
On the older leaves, the edges will look burned, a \\
symptom known as scorch. Plants will easily lodge and \\
be sensitive to disease infestation. Fruit and seed \\
production will be impaired and of poor quality. \\
Excess \\
Plants will exhibit typical Mg, and possibly Ca \\
deficiency symptoms due to a caution imbalance
\end{tabular}

Figure 5 shows the Potassium Deficiency of maize crop leaf and the description about it to the users given by expert system 


\section{International Journal of Science and Research (IJSR) \\ ISSN (Online): 2319-7064}

Index Copernicus Value (2013): 6.14 | Impact Factor (2014): 5.611

Image of Magnesium Deficiency Maize Crop Leaf

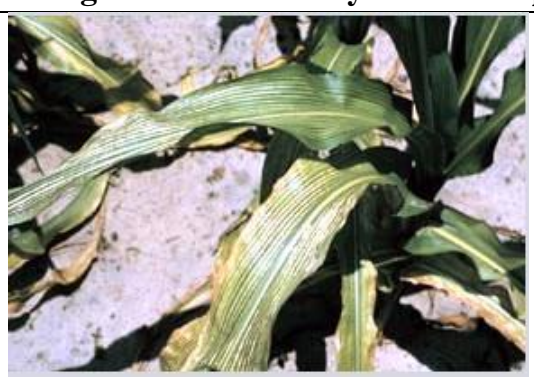

\section{Description}

Deficiency

Older leaves will be yellow in color with interveinal chlorosis (yellowing between the veins) symptoms. Plant growth will be slow and some plants may be easily infested by disease.

Excess

Results in a cation imbalance showing signs of either a Ca or K deficiency.

Figure 6 shows the Magnesium Deficiency of maize crop leaf and the description about it to the users given by expert system

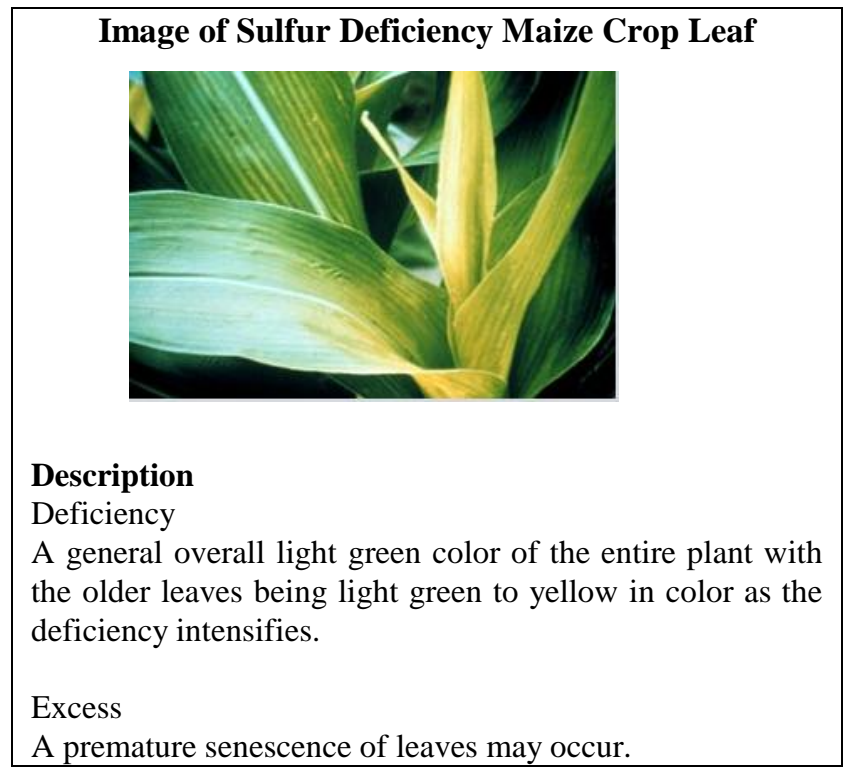

Figure 7 shows the Sulfur Deficiency of maize crop leaf and the description about it to the users given by expert system

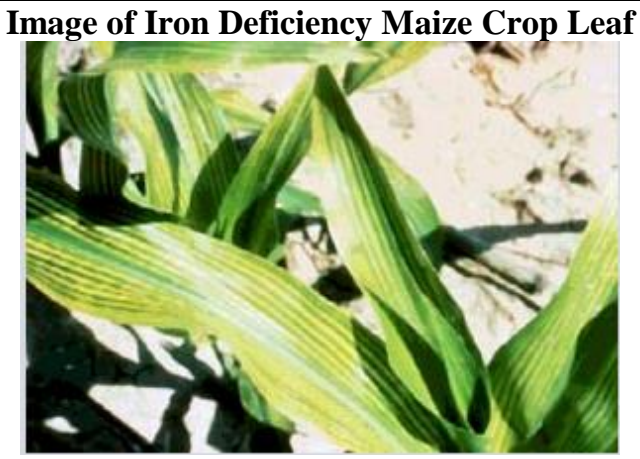

\section{Description}

Deficiency

Interveinal chlorosis will occur on the emerging and young leaves with eventual bleaching of the new growth. When severe, the entire plant may be light green in color.

\section{Excess}

A bronzing of leaves with tiny brown spots on the leaves, a typical symptom frequently occurring with maize crop leaf.

Figure 8 shows the Iron Deficiency of maize crop leaf and the description about it to the users given by expert system

The objectives of the proposed expert system are as follows:

- To develop software which diagnose leaf nutrition deficiency of maize through image processing techniques

- To reinforce through textual input if image is not available.

- To specify remedies for the identified diseases

\section{Conclusion}

Expert Systems can be of great help to the farmers as well as the researchers. Their efficiency of diagnosing the right disease and treatment can enhance the productivity and reduce the losses. Expert systems and decision support systems are widely used in developed countries. This proposed expert system analysis the nutrient deficiency in maize crop leaves using the image processing system and it acts as the advisor to the farmers. Hence this expert-based system was developed to provide immediate attention to the disease infested maize plantation as discovered by the educated agricultural farmers when agricultural extension agents are not present. This would also provide a means to preserve irreplaceable human knowledge expert. It would serve as a quick search for information on how to combat or disinfectant the infested maize plantation in order to get the desired quantity and quality of maize productivity.

\section{References}

[1] New York Times, Tracking the Ancestry of Corn Back 9,000 Years," Retrieve May 25, 2010.

[2] FAO, Maize, rice and wheat: Area harvested, production quantity, and yield," Food and Agriculture Organization (FAO) of the United Nations, Statistics Division, 2009

[3] IITA, UI and IFPRI, Assessment of Nigeria's Agricultural Policy (ANAP): Mitigating Constraints to Commercialization and Investments in Nigeria's Agriculture," Draft Report May 15, implemented by 


\section{International Journal of Science and Research (IJSR) \\ ISSN (Online): 2319-7064}

Index Copernicus Value (2013): 6.14 | Impact Factor (2014): 5.611

International Institute of Tropical Agriculture (IITA) in collaboration with University of Ibadan (UI) and International Food Policy Research Institute (IFPRI) pp.147, 2003.

[4] All India Coordination Research Project (AICRP) on Maize, 2007. 50th Annual Report by Directorate of Maize Research, Indian Council of Agriculture Research (ICAR). pp 6 Pusa, New Delhi

[5] Davenport, H. Thomas and P. Lawrence, Working Knowledge: How Organizations Manage What They Know". Boston: Harvard Business School Press, 1997.

[6] P. Jackson, Introduction to Expert Systems," 3rd edition, New York: Addison-Wesley, ISBN 10-20187686-8, 1998.

[7] K. Darlington, The Essence of Expert Systems', Pearson Education, ISBN 0-13-022774-9, 2000

[8] Shu-Hsien Liao eta. Expert system methodologies and applications - a decade reviews from 1995 to 2004" Expert Systems with Applications 28 (2005) 93-103

[9] Abu- Naser S S, Kashkash K A and Fayyad M (2008) Developing an Expert System for Plant Disease Diagnosis. Journal of Artificial Intelligence 1(2).

[10] Xin J, Zazueta F S and Beck H W (2003) A Web-based Distance Diagnostic and Identification System for Extension. EFITA Conf.

[11] Rafea A, El-Azhari S, Hassan E Integrating Multimedia with Expert Systems for Crop Production Management.

[12] Rafea A Integrating Agricultural Expert Systems with Data Bases and Multimedia.

[13] Rafea A Natural Resources Conservation and Crop Management Expert Systems.

[14] El-Helly M, Rafea A and El-Gammal S (2003) An integrated image processing system for leaf diseases detection and diagnosis. Proc 1st Indian International Conf on AI.

[15] Chaki J and Parekh R (2012) Designing an Automated System for Plant Leaf Recognition. IJAET 2(1):149158.

[16] El-Helly M, Rafea A, El-Gamal S and El-Whab R D Integrating Diagnostic Expert System with Image Processing via Loosely Coupled Technique.

[17] Kumar V, Lehri S, Sharma A K, Meena P D and Kumar A (2008) Image based rapeseed-mustard disease expert system: An effective extension tool. Ind Res J Ext Edu 8.

\section{Author Profile}

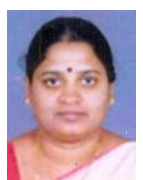

S. Sridevy is a research scholar under Dr. Anna Saro Vijendran. She is currently working as Assistant Professor, Dept. of Physical Sciences \& Information Technology, Agricultural Engineering College \& Research Institute, Tamil Nadu Agricultural University, Coimbatore-641 003, Tamil Nadu, India. Her professional interests are Data Mining, Image Processing and eContent Development.

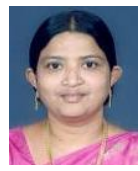

Dr. Anna Saro Vijendran received Ph.D. Degree in Computer Science from Mother Teresa Womens' University, Tamil Nadu, India, in 2009. She has 20 years of experience in teaching. She is currently working as the Director, MCA in SNR Sons College, Coimbatore, Tamil Nadu, India. She has presented and published many papers in International and National conferences. She has authored and co-authored more than 30 refereed papers. Her professional interests are Image Processing, Image fusion, Data mining and Artificial Neural Networks. 